\title{
Novel sulfur hydrides synthesized at extreme conditions
}

\author{
Dominique Laniel $\odot,{ }^{1, *}$ Bjoern Winkler, ${ }^{2}$ Elena Bykova $\odot,{ }^{3}$ Timofey Fedotenko, ${ }^{1}$ Stella Chariton $\odot,{ }^{4}$ Victor Milman $\odot,{ }^{5}$ \\ Maxim Bykov, ${ }^{6}$ Vitali Prakapenka, ${ }^{4}$ Leonid Dubrovinsky $\odot,{ }^{6}$ and Natalia Dubrovinskaia $\odot^{1,7}$ \\ ${ }^{1}$ Material Physics and Technology at Extreme Conditions, Laboratory of Crystallography, University of Bayreuth, 95440 Bayreuth, Germany \\ ${ }^{2}$ Institut für Geowissenschaften, Abteilung Kristallographie, Johann Wolfgang Goethe-Universität Frankfurt, \\ Altenhöferallee 1, D-60438, Frankfurt am Main, Germany \\ ${ }^{3}$ Photon Science, Deutsches Elektronen-Synchrotron, Notkestrasse 85, 22607 Hamburg, Germany \\ ${ }^{4}$ Center for Advanced Radiation Sources, University of Chicago, Chicago, Illinois 60637, USA \\ ${ }^{5}$ Dassault Systèmes BIOVIA, CB4 OWN Cambridge, United Kingdom \\ ${ }^{6}$ Bayerisches Geoinstitut, University of Bayreuth, 95440 Bayreuth, Germany \\ ${ }^{7}$ Department of Physics, Chemistry, and Biology (IFM), Linköping University, SE-581 83, Linköping, Sweden
}

(Received 24 August 2020; revised 29 September 2020; accepted 29 September 2020; published 15 October 2020)

\begin{abstract}
The sulfur-hydrogen system is the first one in which superconductivity at temperatures over $200 \mathrm{~K}$ has been reported, albeit at high pressure. The particular phases causing the measured $T_{c}$ and their structures are not yet firmly identified. Here, synchrotron single-crystal x-ray diffraction studies of S-H samples were performed up to $150 \mathrm{GPa}$ and revealed two previously unobserved and unpredicted sulfur-hydrogen phases- $-\mathrm{H}_{6 \pm x} \mathrm{~S}_{5}$ with $x \sim$ 0.4 , and $\mathrm{H}_{2.85 \pm y} \mathrm{~S}_{2}$ with $y \sim 0.35$. The crystallographic data obtained in this work, both for the new phases and for the previously identified $\mathrm{H}_{3} \mathrm{~S}$ polymorphs, provide an unambiguous experimental proof of the chemical richness of the S-H system and the structural diversity of compounds forming at high pressures and high temperatures. Our results have profound implications for the interpretation of the resistance, superconductivity, and other physical properties measurements on the complex S-H system.
\end{abstract}

DOI: 10.1103/PhysRevB.102.134109

\section{INTRODUCTION}

The search for room-temperature superconductors, those with a superconducting critical temperature $\left(T_{c}\right)$ above $0{ }^{\circ} \mathrm{C}$ $(273 \mathrm{~K})$, has been pursued for decades. With the report of a $T_{c}$ of $203 \mathrm{~K}$ in the S-H system at $150 \mathrm{GPa}$ [1], room-temperature superconductivity transitioned from a distant objective to a realistic endeavor. Although numerous studies over the last several years gave rise to novel metal hydrides [2-11], among which $\mathrm{LaH}_{10}$ has the highest measured $T_{c}$ of $260 \mathrm{~K}$ [12-14], the sulfur-hydrogen system remains the most iconic for modelling, testing, and understanding room-temperature superconductivity.

In the myriad of experiments performed on $\mathrm{S}-\mathrm{H}$ systems, above $40 \mathrm{GPa}$ only sulfur and polymorphs of $\mathrm{H}_{3} \mathrm{~S}$ have been categorically detected on the basis of powder x-ray diffraction (XRD) [15-20]. These $\mathrm{H}_{3} \mathrm{~S}$ polymorphs are thought to follow a sequence of phase transitions: $I 4 / \mathrm{mcm} \underset{\sim 40 \mathrm{GPa}}{\longrightarrow} \mathrm{Cccm}$ $\underset{110 \mathrm{GPa}}{\longrightarrow} R 3 m \underset{150 \mathrm{GPa}}{\longrightarrow} \operatorname{Im}-3 m$ ) [15-19]. Observing exclusively the sulfur-hydrogen compound with the $\mathrm{H}_{3} \mathrm{~S}$ stoichiometry is strikingly at odds with convex hull calculations on the $\mathrm{S}-\mathrm{H}$ system [21,22]. Indeed, whereas in the pressure range of 40-150 $\mathrm{GPa}_{3} \mathrm{~S}$ always appears on the convex hull as the $\mathrm{H}$-richest phase, a variety of phases with a lower hydrogen content, such as $\mathrm{HS}_{2}, \mathrm{H}_{3} \mathrm{~S}_{5}, \mathrm{H}_{5} \mathrm{~S}_{8}, \mathrm{H}_{4} \mathrm{~S}_{3}$, and $\mathrm{H}_{3} \mathrm{~S}_{2}$ [21,22],

*Corresponding author: dominique.laniel@uni-bayreuth.de have been predicted. It is worth noting that $\mathrm{H}_{4} \mathrm{~S}_{3}$ was previously reported to have been observed experimentally from powder x-ray diffraction [21]. Still, the diffraction patterns that were presented contained diffraction lines belonging to a handful of phases, whose strong overlapping prevents an unambiguous interpretation of the data and thus the definitive proof of its formation. Thus univocally demonstrating the presence of a low $\mathrm{H}$ concentration phase could resolve the discrepancy between experiments and calculations, as well as provide an explanation for another enigmatic observation, namely, the large range of $T_{c}$ obtained from resistivity measurements $[1,19]$, nuclear resonance scattering experiments [23], and optical spectroscopy [24].

Computational approaches to search for stable structures of materials using first-principles methods guide experimental investigations, including those of superhydrides [25-27], as they predict the stoichiometry, crystal structure, and some physical properties of hypothetical compounds. Although this synergetic theoretical-experimental approach has undoubtedly yielded impressive results, an independent experimental validation of $a b$ initio predictions is absolutely critical to verify their accuracy. Notably, a full structural solution exclusively dependent on experimental data is still lacking for the high-pressure $\mathrm{H}_{3} \mathrm{~S} \mathrm{Im-3m}$ phase, reported to be superconducting $[1,16,18]$. In order to clarify the existence of $\mathrm{H}_{x} \mathrm{~S}_{y}$ compounds in the S-H system up to $150 \mathrm{GPa}$ and to establish their crystal structures, we have performed synchrotron single-crystal x-ray diffraction (sc-XRDp, described in Refs. [28,29]) on the polycrystalline samples. 
Here we report the synthesis of two sulfur-hydrogen phases, $\mathrm{H}_{6 \pm x} \mathrm{~S}_{5},(x \sim 0.4)$ and $\mathrm{H}_{2.85 \pm y} \mathrm{~S}_{2}(y \sim 0.35)$, with sulfur-to-hydrogen ratios of about $1: 1.2$ and $1: 1.4$, respectively, which have neither been previously observed nor predicted. The structural positions of the heavy sulfur atoms were unambiguously experimentally determined, and $a b$ initio calculations were employed for estimating the amounts and likely positions of hydrogen atoms. Additionally, the structural refinements of the $S$ atomic positions for two previously reported $\mathrm{H}_{3} \mathrm{~S}$ phases, also observed in our experiments, are provided. Our results demonstrate the compositional and structural variety in the S-H system, highlight the importance of precise structural studies, and call for reconsidering the phenomenon of a broad $T_{c}$ variation previously reported in the $\mathrm{S}-\mathrm{H}$ system.

\section{EXPERIMENTAL AND COMPUTATIONAL METHODS}

\section{A. Experimental methods}

Six BX90-type screw diamond anvil cells (DACs) were equipped with diamonds of culet sizes ranging from 250 to $80 \mu \mathrm{m}$, and rhenium was employed as the gasket material. In all cases, an $\mathrm{S}$ piece was embedded in paraffin oil $\left(\mathrm{C}_{\mathrm{n}} \mathrm{H}_{2 \mathrm{n}+2}\right)$, acting as both a pressure-transmitting media as well as a hydrogen reservoir. As showcased in recent works on metal hydrides $[7,9,13,30]$, paraffin and ammonia borane are effective alternatives to pure hydrogen for DAC synthesis experiments. Compared to pure $\mathrm{H}_{2}$, paraffin supplies a reduced amount of hydrogen that should favor the formation of $\mathrm{H}-\mathrm{S}$ solids with lower $\mathrm{H}$ content, which has been predicted but not unambiguously observed so far.

At pressures of $17,45,46,62,100,130$, and $150 \mathrm{GPa}$, the sulfur pieces embedded in paraffin were laser-heated and the resulting chemical reaction products were probed using synchrotron X-ray diffraction. The samples were compressed at ambient temperature. The double-sided YAG laser heating of the samples was performed at the Bayreuth Geoinstitut [31], the P02.2 beamline of PETRA III, and the 13-IDD beamline of the Advanced Photon Source (APS), with sulfur acting as the laser absorber. In all cases, temperatures were measured using the samples' thermal radiation [32]. The pressure inside the sample chamber was determined from the vibrational mode of the stressed diamond anvil and verified by measuring the lattice parameters of the Re gasket at the edge of the experimental chamber [33,34]. The samples were mainly characterized by single-crystal (sc-XRDp) and powder $\mathrm{X}$-ray diffraction (XRDp) measurements. The subscript "p" in sc-XRDp emphasizes the very small, just a few microns size of the single crystals, for which a special approach to the high-pressure XRD data acquisition and analysis was recently developed in high-pressure crystallography [28], and its reliability demonstrated numerous times [35-37]. The x-ray diffraction data were acquired at the P02.2 and 13-IDD beamlines of PETRA III and the APS, respectively. More details on the experimental procedure can be found in the Supplemental Material [38].

\section{B. Computational methods}

Density functional theory (DFT) calculations have been performed using the CASTEP code [39]. The code is an implementation of Kohn-Sham DFT based on a plane-wave basis set in conjunction with pseudopotentials. The planewave basis set is unbiased (as it is not atom-centered) and does not suffer from the problem of basis-set superposition errors, unlike atom-centered basis sets. It also makes converged results straightforward to obtain in practice, as the basis set convergence is controlled by a single adjustable parameter, the plane-wave cutoff. Pseudopotentials were either norm conserving or ultrasoft and were generated using the Perdew-Burke-Ernzerhof (PBE) exchange-correlation functional [40] using the "on-the-fly" parameters included in the CASTEP 2019 distribution. These pseudopotentials have been shown to be very accurate and are very well suited for the calculations carried out here [41]. The Brillouin-zone integrals were performed using Monkhorst-Pack grids [42] with spacings between grid points of less than $0.02 \AA^{-1}$. Full geometry optimizations of the unit-cell parameters and the internal coordinates were performed until forces were typically converged to $<0.005 \mathrm{eV} / \AA$, although sometimes this criterion was relaxed in order to save computing resources, and the residual stress was $<0.02 \mathrm{GPa}$.

In our calculations, temperature, configurational entropy, and the entropy contribution due to lattice vibrations were neglected. Force and stress-free configurations obtained for a specific atomic configuration and a preset pressure correspond to local minima (metastable phases) or the global minimum (thermodynamically stable phase) of the enthalpy landscape. Further details on the computational method are found in the Supplemental Materials [38].

\section{RESULTS}

\section{A. Previously unobserved S-H phases}

At $17 \mathrm{GPa}$, the sulfur piece quickly melted at about $1500 \mathrm{~K}$, in agreement with the melting curve of sulfur under pressure [43], and its prolonged heating was not possible. X-ray diffraction mapping of the sample after heating revealed no sign of a chemical reaction between sulfur and hydrogen, likely due to an insufficient decomposition of paraffin oil and thus very limited hydrogen supply. However, laser heating of the sulfur-paraffin samples to about $2000 \mathrm{~K}$ in the pressure interval of 45-130 GPa resulted in the appearance of new reflections in the diffraction patterns, which could be indexed with a tetragonal unit cell (space group $I 4_{1}$, no. 80). At $62 \mathrm{GPa}$, the unit-cell parameters were found to be $a=$ $13.4935(4) \AA$ and $c=11.5793(7) \AA\left[V=2108.296(14) \AA^{3}\right]$. High-quality single-crystal XRD data acquired at this pressure allowed the accurate determination and refinement of the sulfur atomic coordinates. The unit cell of the new structure contains 140 sulfur atoms-hereafter named $t I 140$ - on 19 crystallographically independent positions ( $8 b$ and $4 a$ ) (see Tables I and S1 [38]). The unit-cell volume per sulfur atom in $t I 140$ is equal to $15.1 \AA^{3}$ at $62 \mathrm{GPa}$, significantly larger than in the structure of pure sulfur $\left(11.6 \AA^{3}\right)$ at the same pressure. This strongly suggests the presence of hydrogen in the structure of $t I 140$.

As seen in Fig. 1, the main structural elements of $t I 140$ are sinusoidal $\mathrm{S}$ chains with a period of six atoms, going along the $a$ and $b$ directions. Each sulfur atom located at the minimum 
TABLE I. Selected crystallographic data for pure $S, t I 140, h P 112, I 4 / m c m \mathrm{H}_{3} \mathrm{~S}$ and $I m-3 m \mathrm{H}_{3} \mathrm{~S}$. The full crystallographic data for each phase and the pressures at which they have been observed are provided in the Supplemental Materials [38]. Aside from pure sulfur, the sulfur-hydrogen phases are named in analogy with the Pearson notation. The two letters specify the Bravais lattice, but the number only gives the amount of sulfur atoms in the unit cell, as the number of hydrogen atoms cannot be determined experimentally.

\begin{tabular}{|c|c|c|c|c|c|}
\hline & $S$ & $t I 140$ & $h P 112$ & $t I 8, \mathrm{H}_{3} \mathrm{~S}$ & $c I 2, \mathrm{H}_{3} \mathrm{~S}$ \\
\hline Pressure (GPa) & 46 & 62 & 150 & 100 & 150 \\
\hline Space group (no.) & $I 4_{1} /$ acd $(142)$ & $I 4_{1}(80)$ & $P-3 c 1(165)$ & I4/mcm (140) & Im-3m (229) \\
\hline$b(\AA)$ & $7.9281(19)$ & $13.4935(4)$ & $8.9840(7)$ & $5.4513(3)$ & $3.076(4)$ \\
\hline$c(\AA)$ & $3.1856(8)$ & $11.5793(7)$ & $19.283(2)$ & $4.5825(3)$ & $3.076(4)$ \\
\hline$\gamma\left({ }^{\circ}\right)$ & 90 & 90 & 120 & 90 & 90 \\
\hline$R_{\mathrm{int}}$ & 0.053 & 0.0546 & 0.0347 & 0.0747 & 0.1259 \\
\hline$R_{1}$ & 0.0430 & 0.0519 & 0.0335 & 0.0409 & 0.0583 \\
\hline$w R_{2}$ & 0.0464 & 0.0590 & 0.0740 & 0.0448 & 0.0770 \\
\hline$R_{1}$ (all data) & 0.0474 & 0.0626 & 0.0399 & 0.0473 & 0.0583 \\
\hline$w R_{2}$ (all data) & 0.0472 & 0.0628 & 0.0771 & 0.0460 & 0.0770 \\
\hline
\end{tabular}

and at the maximum of the oscillating chain is bound to a third $\mathrm{S}$ atom acting as a linker to a neighboring chain. As dictated by the $I 4_{1}$ space group's $4_{1}$ symmetry operation, the chains at each $1 / 4 c$ increment are perpendicular to each other. Within these chains, first-neighbor sulfur contacts range between 2.0560(18) and 2.4799(18) $\AA$ at $62 \mathrm{GPa}$. The shortest interatomic distance between $\mathrm{S}$ atoms of different chains or layers is $\sim 2.9 \AA$ or longer. Adopting peculiar chain arrangements is typical of sulfur allotropes, as they are also observed in its S-II and S-III phases [44].

Laser heating of the sulfur-paraffin sample at $150 \mathrm{GPa}$ resulted in the synthesis of another hitherto unknown phase. The analysis of the single-crystal XRD data gave a large hexagonal unit cell (space group $P-3 c 1$, no. 165) with lattice parameters

(a)

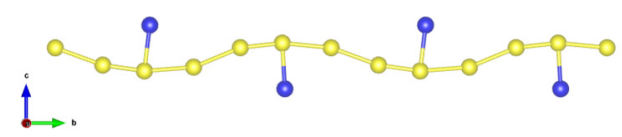

(b)

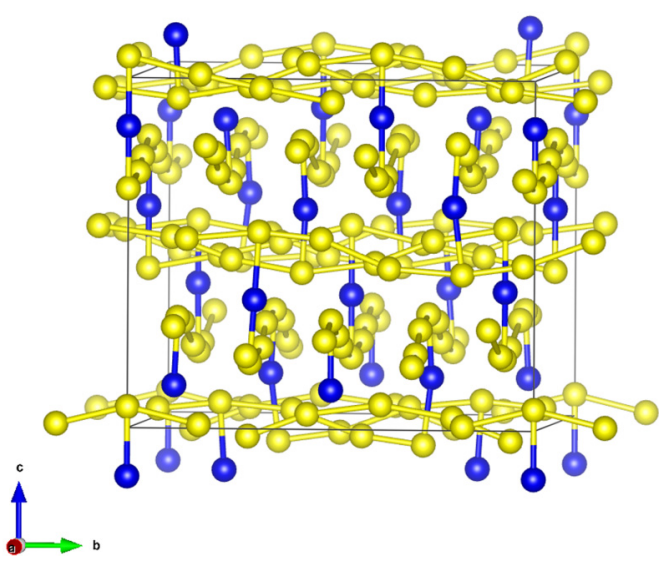

FIG. 1. Structure of the $t I 140$ compound (hydrogen atoms not shown). (a) A single sinusoidal strand of $\mathrm{S}$ atoms. (b) The $t I 140$ unit cell. Yellow spheres represent sulfur atoms forming sinusoidal chains, while the blue spheres are sulfur atoms linking the chains together. $a=8.9840(7) \AA$ and $c=19.283(2) \AA\left[V=1347.9(2) \AA^{3}\right]$. The cell contains 112 sulfur atoms occupying 12 independent crystallographic positions (see Tables I and S4 [38]). Henceforth, this phase is referred to as $h P 112$. The unit-cell volume per sulfur atom in $h P 112$ is equal to $12.0 \AA^{3}$ at $150 \mathrm{GPa}$, which is larger than that in pure sulfur $\left(8.7 \AA^{3}\right)$ at the same pressure, again suggesting the synthesis of a sulfur-hydrogen compound.

Its complex crystal structure (Fig. 2) can be described as a stacking of nets of sulfur atoms (represented by yellow spheres in Fig. 2) with the slightly distorted 3.6.3.6 tessellation. Lying in the $a b$ planes and stacked along the $c$ axis, the nets form triangular and hexagonal channels populated by sulfur atoms (green and orange spheres in Fig. 2). In the distorted hexagons, the S-S interatomic distances vary from $2.0140(15)$ to $2.5710(15) \AA$. The $\mathrm{S}$ atoms in the hexagonal channels (shown orange in Fig. 2) are separated by distances of 1.963(2)-2.4924(13) $\AA$. The S atoms in the triangular channels (shown green in Fig. 2) are located much further apart from one another, at distances of $\sim 5.0 \AA$ along the $c$ axis, similar to the gap between the tessellated nets $(\sim 4.6 \AA)$. If the distortions of the nets are neglected, then the $h P 112$ structure can be described with a higher symmetry subcell $(P 6 / \mathrm{mmm}$ space group; $a / 2, c / 4)$, as detailed in the Supplemental Material [38], Fig. S5.

The $t I 140$ and $h P 112$ structures derived from the analysis of single-crystal XRD data were verified not to contain carbon atoms, which could potentially originate from the diamond anvil or the decomposed paraffin oil. Indeed, no $\mathrm{S}$ atom could be substituted with a $\mathrm{C}$ atom, and no significant electronic density residuals at crystal chemically reasonable distances for an S-C bond were observed. In the XRD patterns at relevant pressures and temperatures, the diffraction of nanocrystalline diamond was observed, inferring that it is, along with $\mathrm{H}$, a decomposition of paraffin oil. Also, the unitcell, space group, and sulfur atom arrangement of the $t I 140$ and $h P 112$ compounds were compared against the H-S solids previously theoretically predicted, including those identified as potential decomposition products of $\mathrm{H}_{2} \mathrm{~S}[21,22]$, and no match was found. 
(a)

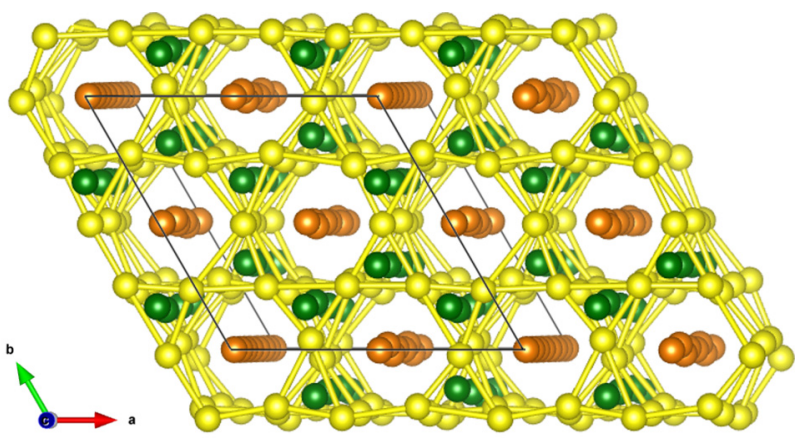

(b)

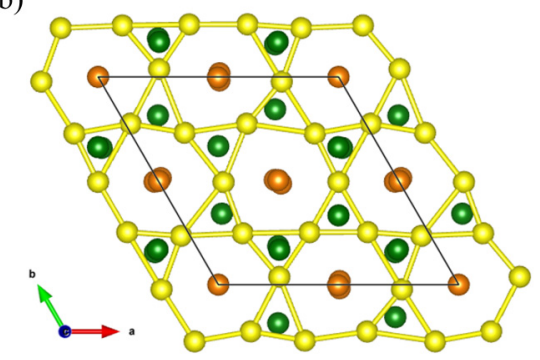

(c)

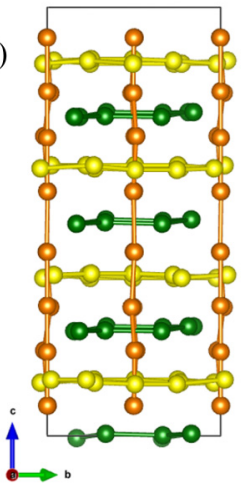

FIG. 2. Structure of the $h P 112$ compound (hydrogen atoms are not shown): (a) a prospective view with the unit cell outlined; (b) a projection along the $c$ axis; and (c) a projection along the $a$ axis. Sulfur atoms forming the slightly distorted 3.6.3.6 nets stacking along the $c$ axis are shown in yellow, those filling the triangular channels in green, and those filling the hexagonal channels in orange.

\section{B. Hydrogen content and stability of the $t I 140$ and $h P 112$ phases}

As shown in Fig. 3, the volume per sulfur atom for the $t I 140$ and $h P 112$ phases obtained here are in-between those of pure sulfur and of $\mathrm{H}_{3} \mathrm{~S}$, as well as very close to that of $\mathrm{H}_{4} \mathrm{~S}_{3}$, thus pointing towards the incorporation of hydrogen into their structure. However, experimentally locating the position of hydrogen atoms by x-ray diffraction in high-pressure compounds is notoriously difficult due to the restrictions inherent to DAC experiments. Thus, in order to predict from DFT calculations the concentration and possible location of hydrogen atoms in the $t I 140$ and $h P 112$ phases, the experimentally determined sulfur positions, space group symmetry, and unitcell dimensions were exploited. It is very well established that DFT-based models can predict the position of hydrogen atoms reliably (e.g., Ref. [45]).

The approach chosen here was to use the experimentally determined lattice parameters, space group symmetry, and sulfur atomic positions. Then, voids suitable for hosting hydrogen atoms were searched for by computing Connolly surfaces. With this approach it cannot be shown conclusively that all possible hydrogen locations were found, but as discussed below, it provides robust information on the probable hydrogen concentration of the compounds. The likely concentration is far below the maximum numbers of possible hydrogen locations, and hence it is not really relevant if a site was missed.

For each of the two novel high-pressure S-H compounds, the structural parameters were computed first with no

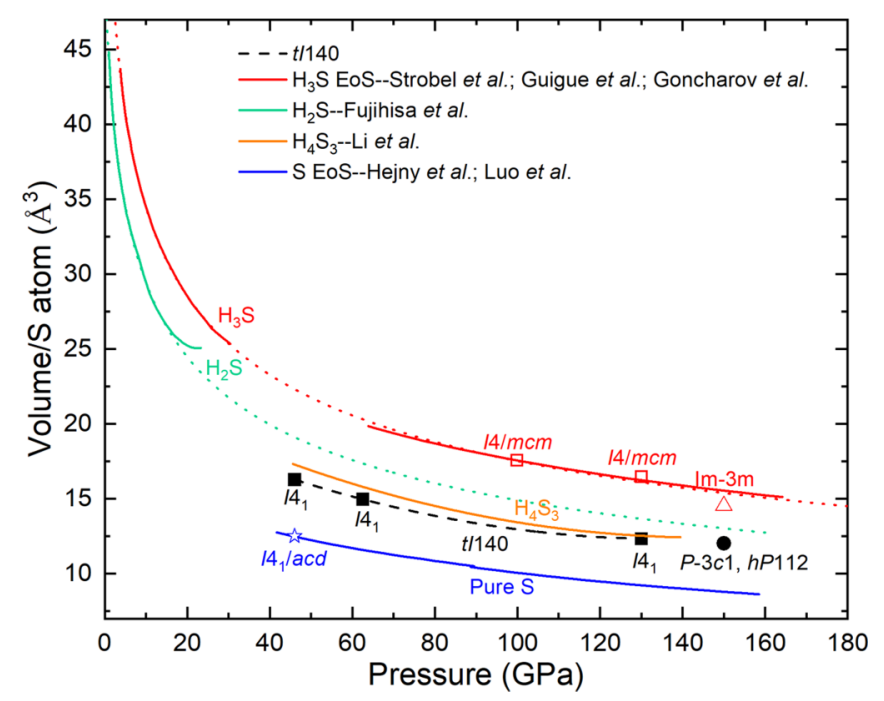

FIG. 3. Unit-cell volume per sulfur atom as a function of pressure for $\mathrm{H}_{3} \mathrm{~S}$ (full red line comprising the $I 4 / \mathrm{mcm}, \mathrm{Cccm}, \mathrm{R} 3 \mathrm{~m}$, and Im-3m phases) [15,17,46], $\mathrm{H}_{2} \mathrm{~S}$ (full green line) [47], $\mathrm{H}_{4} \mathrm{~S}_{3}$ (full orange line) [21], $t I 140, h P 112$ (full black symbols), and pure $\mathrm{S}$ (fill blue line and symbol) $[48,49]$. The empty red symbols $(I 4 / \mathrm{mcm}$ and $I m-3 m)$ are from our experiments. The red and green dotted lines are the extrapolations of the equations of state of $\mathrm{H}_{3} \mathrm{~S}$ [46] and $\mathrm{H}_{2} \mathrm{~S}$ [47], respectively. The $\mathrm{H}_{2} \mathrm{~S}$ solid is known to disproportionate between about 20 and $46 \mathrm{GPa}$ [22]. The dashed black line is a guide to the eye.

hydrogen incorporated and then calculated for a set of compounds with increasing hydrogen content. For the $t I 140$ structure at $62 \mathrm{GPa}, 28$ Wyckoff sites which could be populated by $\mathrm{H}$ atoms were found, yielding $\mathrm{H}_{224} \mathrm{~S}_{140}$ as the most hydrogen-rich composition. For the hP112 structure, 288 positions were found, meaning that the most hydrogen-rich composition is $\mathrm{H}_{288} \mathrm{~S}_{112}$. The results shown in Fig. 4 demonstrate that the unit-cell volume of both $t I 140$ and $h P 112$ is essentially independent of the actual atomic configuration but mostly depends on the composition. Not only is the unitcell volume of sulfur-V [50] close to that of hypothetical hydrogen-free $h P 112$, but the volumes of the fully ordered compounds, i.e., monoclinic $\mathrm{H}_{3} \mathrm{~S}_{5}$ and tetragonal $\mathrm{H}_{3} \mathrm{~S}$ [16], are not far off the trend derived here. The unit-cell volume of different configurations for the same hydrogen concentration may differ by up to $15 \AA^{3}$, but this corresponds to $\sim 1 \%$ only and is less than the symbol size. This then allowed a robust estimate of the actual hydrogen concentration of the compounds experimentally observed, and hence it is concluded that the most likely composition for the $t I 140$ phase at $62 \mathrm{GPa}$ is $\mathrm{H}_{6 \pm x} \mathrm{~S}_{5}$, with $x \sim 0.4$, and the most likely composition of the $h P 112$ phase at $150 \mathrm{GPa}$ is $\mathrm{H}_{2.85 \pm y} \mathrm{~S}_{2}$, with $y \sim 0.35$.

The compounds which were synthesized are therefore best described in analogy to the well-established interstitial carbides and nitrides. These are structures where the large metal provides a matrix, and the small carbon or nitrogen enters interstitial sites, with a very broad compositional range. This is exactly what the model calculations predict here: a matrix due to the polymerized sulfur atoms, and the interstitial sites partially occupied by hydrogen. For a given concentration and 
(a)

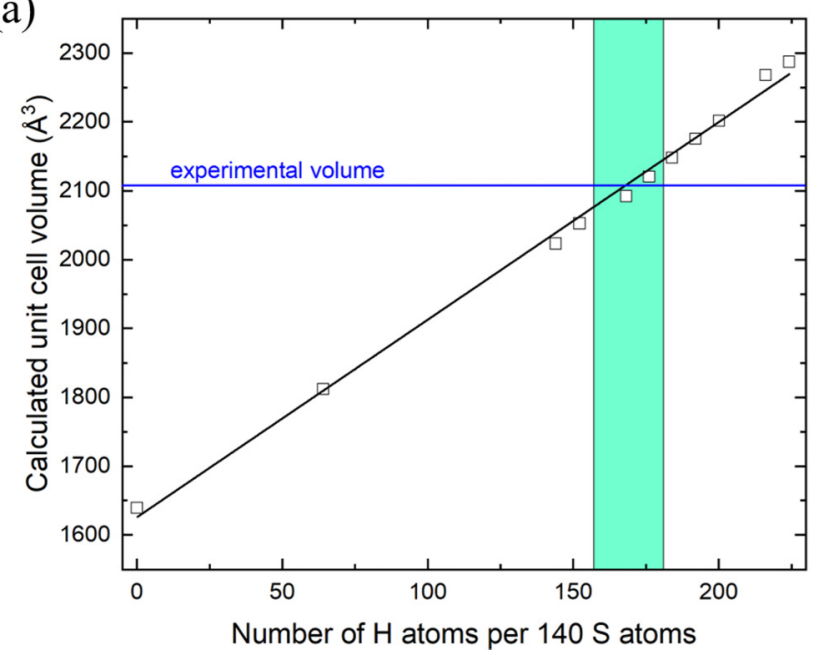

(b)

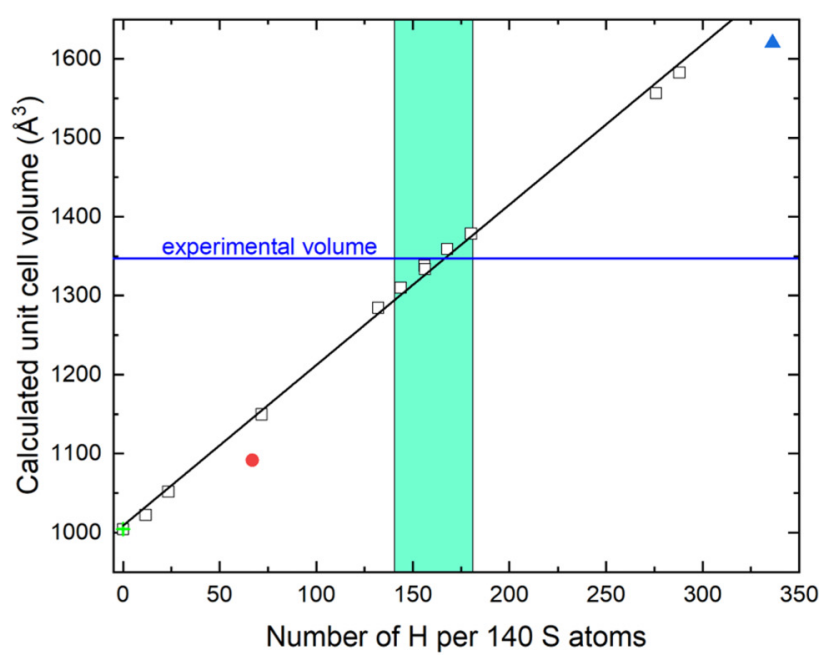

FIG. 4. (a) Predicted unit-cell volumes as a function of hydrogen content for the $t I 140$ structure at 62 GPa. (b) Predicted unit-cell volumes as a function of hydrogen content for the $h P 112$ structure at $150 \mathrm{GPa}$ (empty black squares), volume of sulfur-V (green cross) [50], monoclinic $\mathrm{H}_{3} \mathrm{~S}_{5}$ (red circle) [16], and tetragonal $\mathrm{H}_{3} \mathrm{~S}$ (blue triangle) [16]. Cyan boxes indicate the most likely composition of the experimentally investigated samples.

pressure, the computations reveal that many structures (i.e., with different $\mathrm{H}$ position) are energetically degenerate.

Typical structures obtained for the $\mathrm{H}_{6 \pm x} \mathrm{~S}_{5}(x \sim 0.4$ at 62 GPa) $t I 140$ and the $\mathrm{H}_{2.85 \pm y} \mathrm{~S}_{2}(y \sim 0.35$ at $150 \mathrm{GPa}) h P 112$ are shown in Fig. 5. In $t I 140$ at $62 \mathrm{GPa}$ and $h P 112$ at 150 GPa, S-H contacts increase from 1.37 and $1.39 \AA$ upwards, respectively. A Mulliken population analysis shows that these are covalent bonds, with Mulliken populations decreasing monotonically with increasing S-H distance from $0.72 \mathrm{e} / \AA^{3}$ at $1.37 \AA$ to $0.4 \mathrm{e} / \AA^{3}$ at $1.60 \AA$ for $t I 140$, and from $0.67 \mathrm{e} / \AA^{3}$ at $1.39 \AA$ to $0.52 \mathrm{e} / \AA^{3}$ at $1.46 \AA$ for $h P 112$. It is expected that in the structures observed, a significant part of the voids remains unfilled. Changes in the distribution of the hydrogen atoms for a given composition only leads to small changes of up to a few $10 \mathrm{meV} /$ atom, but generally much less. Hence the structures are most likely disordered. This implies that both $t I 140$ and $h P 112$ are stabilized by a substantial configurational entropy contribution. These compounds are not stoichiometric and their hydrogen concentration is very likely variable as a function of pressure, temperature, hydrogen fugacity, etc.

According to our calculations (Fig. S3), the $h P 112$ phase is more stable than a mechanical mixture of sulfur- $\mathrm{V}$ and hydrogen-III at $150 \mathrm{GPa}[50,51]$. Without accounting for thermal and configurational entropy contributions, $h P 112$ is, however, less stable than $\mathrm{S}+\mathrm{H}_{3} \mathrm{~S}$ (by $\sim 40 \mathrm{meV} /$ atom) at this same pressure. It therefore might only be metastable and could be formed either when the synthesis commences from the elements or due to the decomposition of hydrogen-rich starting materials.

\section{Single-crystal validation of previously observed S-H phases}

Along with the $t I 140$ and $h P 112$ phases, the previously known tetragonal $[15,16,46]$ and cubic [17-19] polymorphs of $\mathrm{H}_{3} \mathrm{~S}$ were observed. We solved the structure and refined the atomic positions of sulfur atoms in the tetragonal $\mathrm{H}_{3} \mathrm{~S}$ phase $(I 4 / \mathrm{mcm}$ space group, $t I 8)$ from single-crystal XRD data at $100 \mathrm{GPa}$. The lattice parameters at this pressure were found to be $a=5.4513(11) \AA$ and $c=4.5825(3) \AA[V=$ 140.71(3) $\AA^{3}$ ], as seen in Tables I and S6 [38]. These lattice parameters and the structural arrangement of the $\mathrm{S}$ atoms match those of the $t I 8 I 4 / \mathrm{mcm} \mathrm{H}_{3} \mathrm{~S}$ phase previously observed $[15,46]$. It was reported that at higher pressures, at least above $100 \mathrm{GPa}$, the $t 18 \mathrm{I} / \mathrm{mcm} \mathrm{H}_{3} \mathrm{~S}$ phase transforms into an orthorhombic (space group Cccm) solid [15-17,20,52]. However, no sign of an orthorhombic distortion was detected here, both from the accurately determined lattice parameters or from the analysis of reciprocal space reconstructions at 100 (see Fig. S6 [38]) and $130 \mathrm{GPa}$.

At $150 \mathrm{GPa}$, analysis of the sc-XRDp measurements showed the presence of the previously known cubic $\mathrm{H}_{3} \mathrm{~S}$ polymorph (space group Im-3m, cI2, Tables I and S8 [38]), whose structure was originally inferred from powder $\mathrm{x}$-ray diffraction data in combination with theoretical calculations [15-17]. Our sc-XRDp data allowed the structure solution and the refinement of the positions of the $\mathrm{S}$ atoms. The lattice parameters display no sign of a trigonal distortion that would be indicative of the rhombohedral $R 3 \mathrm{~m}$ phase-predicted by theory to be stable up to $180 \mathrm{GPa}$ [53] —as demonstrated in Fig. S7 [38].

\section{DISCUSSION}

The results presented highlight the unique capabilities of sc-XRDp in high-pressure crystallography. The rich singlecrystal x-ray diffraction patterns of structurally complex phases with large unit cells-producing a quantity of reflections proportional to their unit-cell size-are easier to untangle due to the possibility to visualize the threedimensional reciprocal space (see Fig. S8 [38]). In contrast, powder XRD data of phases with large unit cells are typically hard to analyze because of the peak overlap and, often, a poor signal-to-noise ratio. As a result, even though previous x-ray diffraction investigations denoted "parasitic" reflections, the structural details of such phases could not be experimentally 

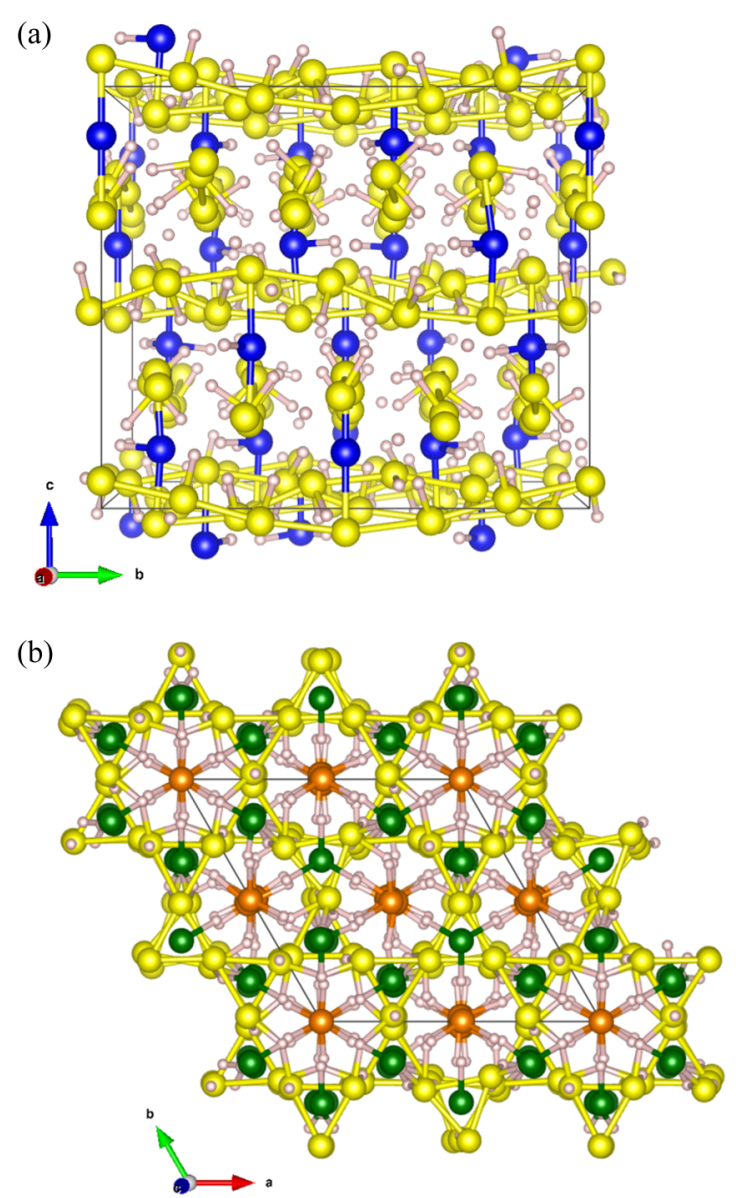

FIG. 5. Representative structures for the $t I 140$ (a) and $h P 112$ (b) compounds, including hydrogen atoms, as derived based on DFT calculations. For both structures just one of the many energetically degenerate configurations of $\mathrm{H}$ atoms is shown. The $\mathrm{H}$ atoms are drawn as pink spheres, whereas all other spheres represent $\mathrm{S}$ atoms.

resolved [16-18,21]. This point can be illustrated if one considers a powder x-ray diffraction pattern of a sample containing both the $c I 2 I m-3 m \mathrm{H}_{3} \mathrm{~S}$ and the $h P 112$ phases (Fig. 6). In this diffractogram, the higher symmetry and smaller unit cell $c I 2 \mathrm{Im}-3 m \mathrm{H}_{3} \mathrm{~S}$ solid is clearly dominating the $\mathrm{X}$-ray diffraction pattern, while the $h P 112$ structure, with a much larger unit cell and lower symmetry, is almost undiscernible.

In addition, the finding and the structural characterization of the $t I 140$ and $h P 112$ compounds point towards the limitations of the $a b$ initio computational approaches to discover stable structures of materials. Indeed, compounds with very large unit cells, like $t I 140$ and $h P 112$, would fall outside of the range typically explored in structure searches. In fact, for these partially disordered compounds, it currently is impractical to explore their stability in detail, as this would require very extensive computational resources. It is worth noting that the $\mathrm{H}_{4} \mathrm{~S}_{3}$ phase previously reported [21] was not observed in our experiments.

The formation of the $t I 140$ and $h P 112$ compounds with variable stoichiometry in the pressure range of 45-130 GPa and at $150 \mathrm{GPa}$, respectively, has profound implications for the understanding of the S-H phase diagram and, in turn, for the analysis and interpretation of the resistance as well

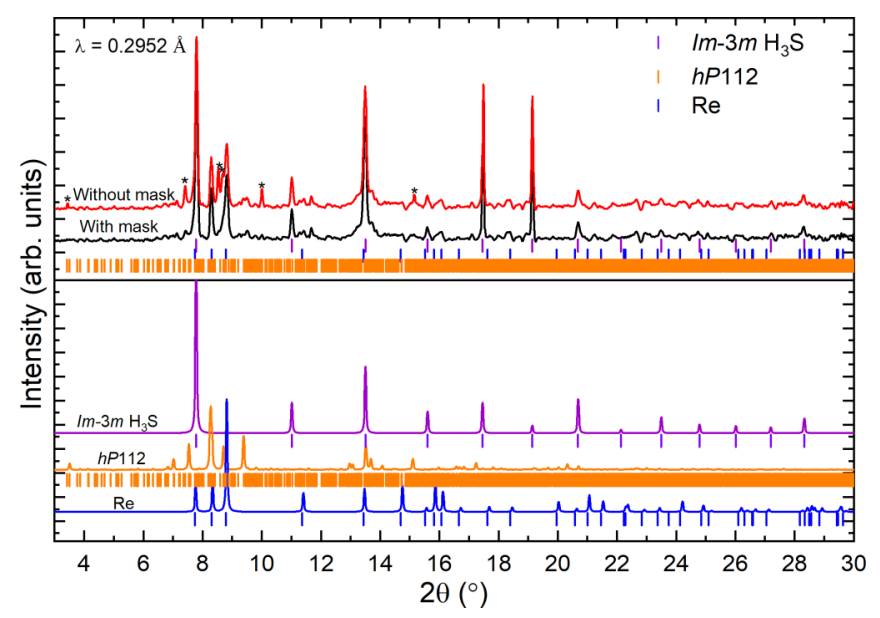

FIG. 6. Upper panel: Powder x-ray diffraction pattern of the sample containing both the $h P 112$ and $c I 2 I m-3 m \mathrm{H}_{3} \mathrm{~S}$ phases. The pattern (red curve) was obtained through integration of the raw twodimensional (2D) XRD data recorded without sample rotation; the $h P 112$ phase in the raw data was manifested by dots of single-crystal reflections only-their positions in the integrated red pattern are marked by asterisks. Masking the single-crystal reflections (see Fig. S9 [38]) before the integration makes the $h P 112$ phase unidentifiable (black curve). Bottom panel: Calculated diffractograms of the $c I 2$ Im- $3 m \mathrm{H}_{3} \mathrm{~S}, h P 112$, and Re phases (purple, orange, and blue, respectively). Relative intensities of the $h P 112$ peaks in the calculated pattern do not match those in the red diffractogram of the upper panel, as the latter are affected by the nature of the sample-single crystals of the $h P 112$ phase are insufficient in number to produce the diffraction rings - and by the procedure of the experimental data collection (the 2D XRD data were recorded without sample rotation).

as other physical properties measurements on the complex $\mathrm{S}-\mathrm{H}$ system at high pressure $[1,19,23]$. Employed first as a precursor to produce samples reported to have a high $T_{c}$, $\mathrm{H}_{2} \mathrm{~S}$ is known to decompose upon compression to pressures above $\sim 50 \mathrm{GPa}$ [54], yielding $\mathrm{H}_{3} \mathrm{~S}$ as well as phases with a lower $\mathrm{H}$ concentration. However, so far the high $T_{c}$ was attributed solely to the $c I 2 I m-3 m \mathrm{H}_{3} \mathrm{~S}$ phase [17-19], and the other decomposition product was typically assumed to be pure sulfur [18] - contradicting many theoretical predictions $[16,21,22,55]$. The $t I 140$ and $h P 112 \mathrm{~S}-\mathrm{H}$ compounds might be reasonable candidates as the decomposition products of compressed $\mathrm{H}_{2} \mathrm{~S}$, which could potentially explain the variations in its $T_{c}$ measurements [1,23]. The formation of the $t I 140$ and $h P 112$ phases could also be expected from an elemental synthesis $[15,17,19]$. When the $\mathrm{H}: \mathrm{S}$ ratio in the whole sample (i.e. with all atoms in the pressure chamber counted) is lower than 3 , the formation of $t I 140$ and $h P 112$ is anticipated. Even for samples with a $\mathrm{H}: \mathrm{S}$ ratio above 3, sufficient hydrogenation of the bulk of the piece of sulfur is still not ensured, as suggested by "parasitic" $\mathrm{x}$-ray diffraction peaks accompanying those of $\mathrm{H}_{3} \mathrm{~S}[15,17]$.

\section{CONCLUSION}

The presented investigation of the sulfur-paraffin system up to $150 \mathrm{GPa}$ by sc-XRDp led to the synthesis of two new sulfurhydrogen compounds, $t I 140 \mathrm{H}_{6 \pm x} \mathrm{~S}_{5}(x \sim 0.4$ at $62 \mathrm{GPa})$ and 
$h P 112 \mathrm{H}_{2.85+y} \mathrm{~S}_{2}(y \sim 0.35$ at $150 \mathrm{GPa})$. The structure of two previously identified $\mathrm{H}_{3} \mathrm{~S}$ polymorphs $(t I 8 \mathrm{I} / \mathrm{mcm}$ and $c I 2$ $I m-3 m)$ were solved and refined from sc-XRDp data. The very large unit cells of both $t I 140$ and $h P 112$ phases readily explain why neither have been observed in earlier powder x-ray diffraction studies nor predicted by theoretical calculations. The new compounds are not stoichiometric, and their hydrogen concentration is very likely variable as a function of pressure, temperature, hydrogen fugacity, etc.

The discovery of the S-H compounds with low $\mathrm{H}$ concentration firmly reconciles ab initio predictions with experimental observations. These phases provide a key piece in understanding the contentious $\mathrm{H}_{2} \mathrm{~S}$ decomposition products. It is important to investigate whether $t I 140$ and $h P 112$ are superconducting, and if so, at which temperature, because the presence of these phases may affect $T_{c}$ measurements in the H-S system. Our study underlines the importance of such a highly accurate and sensitive characterization method as sc-XRDp for studying complex systems and reveals the difficulties theoretical calculations face in predicting hydrogen-containing high-pressure phases.

\section{ACKNOWLEDGMENTS}

The authors acknowledge the Deutsches ElektronenSynchrotron (DESY, PETRA III) and the Advanced Photon Source (APS) for provision of beamtime at the P02.2 and 13-IDD beamlines, respectively. D.L. thanks the Alexander von Humboldt Foundation for financial support. N.D. and L.D. thank the Federal Ministry of Education and Research, Germany (BMBF, Grant No. 05K19WC1), and the Deutsche Forschungsgemeinschaft (DFG projects DU 954-11/1, DU 393-9/2, and DU 393-13/1) for financial support. B.W. gratefully acknowledges funding by the DFG in the framework of the research unit DFG FOR2125 and within projects WI1232. N.D. thanks the Swedish Government Strategic Research Area in Materials Science on Functional Materials at Linköping University (Faculty Grant No. SFO-Mat-LiU No. 2009 00971).
[1] A. P. Drozdov, M. I. Eremets, I. A. Troyan, V. Ksenofontov, and S. I. Shylin, Nature (London) 525, 73 (2015).

[2] C. M. Pépin, G. Geneste, A. Dewaele, M. Mezouar, and P. Loubeyre, Science, 357, 382 (2017).

[3] X. Li, X. Huang, D. Duan, C. J. Pickard, D. Zhou, H. Xie, Q. Zhuang, Y. Huang, Q. Zhou, B. Liu, and T. Cui, Nat. Commun. 10, 3461 (2019).

[4] J. Binns, M. Peña-Alvarez, M.-E. Donnelly, E. Gregoryanz, R. T. Howie, and P. Dalladay-Simpson, Engineering 5, 505 (2019).

[5] C. M. Pépin, A. Dewaele, G. Geneste, P. Loubeyre, and M. Mezouar, Phys. Rev. Lett. 113, 265504 (2014).

[6] A. Marizy, G. Geneste, P. Loubeyre, B. Guigue, and G. Garbarino, Phys. Rev. B 97, 184103 (2018).

[7] D. V. Semenok, A. G. Kvashnin, A. G. Ivanova, V. Svitlyk, V. Y. Fominski, A. V. Sadakov, O. A. Sobolevskiy, V. M. Pudalov, I. A. Troyan, and A. R. Oganov, Mater. Today 33, 36 (2020).

[8] N. P. Salke, M. M. Davari Esfahani, N. Yedukondalu, Y. Zhang, I. A. Kruglov, J. Zhou, E. Greenberg, V. B. Prakapenka, J. Liu, A. R. Oganov, and J. Lin, Inorg. Chem. 59, 5303 (2020).

[9] D. Zhou, D. V. Semenok, D. Duan, H. Xie, W. Chen, X. Huang, X. Li, B. Liu, A. R. Oganov, and T. Cui, Sci. Adv. 6, eaax6849 (2020).

[10] M. Peña-Alvarez, J. Binns, A. Hermann, L. C. Kelsall, P. Dalladay-Simpson, E. Gregoryanz, and R. T. Howie, Phys. Rev. B 100, 184109 (2019).

[11] B. Guigue, A. Marizy, and P. Loubeyre, Phys. Rev. B 102, 014107 (2020).

[12] Z. M. Geballe, H. Liu, A. K. Mishra, M. Ahart, M. Somayazulu, Y. Meng, M. Baldini, and R. J. Hemley, Angew. Chem. Int. Ed. 57, 688 (2018).

[13] M. Somayazulu, M. Ahart, A. K. Mishra, Z. M. Geballe, M. Baldini, Y. Meng, V. V. Struzhkin, and R. J. Hemley, Phys. Rev. Lett. 122, 027001 (2019).
[14] A. P. Drozdov, P. P. Kong, V. S. Minkov, S. P. Besedin, M. A. Kuzovnikov, S. Mozaffari, L. Balicas, F. F. Balakirev, D. E. Graf, V. B. Prakapenka, E. Greenberg, D. A. Knyazev, M. Tkacz, and M. I. Eremets, Nature (London) 569, 528 (2019).

[15] B. Guigue, A. Marizy, and P. Loubeyre, Phys. Rev. B 95, 020104(R) (2017).

[16] A. F. Goncharov, S. S. Lobanov, I. Kruglov, X. M. Zhao, X. J. Chen, A. R. Oganov, Z. Konôpková, and V. B. Prakapenka, Phys. Rev. B 93, 174105 (2016).

[17] A. F. Goncharov, S. S. Lobanov, V. B. Prakapenka, and E. Greenberg, Phys. Rev. B 95, 140101 (2017).

[18] M. Einaga, M. Sakata, T. Ishikawa, K. Shimizu, M. I. Eremets, A. P. Drozdov, I. A. Troyan, N. Hirao, and Y. Ohishi, Nat. Phys. 12, 835 (2016).

[19] H. Nakao, M. Einaga, M. Sakata, M. Kitagaki, K. Shimizu, S. Kawaguchi, N. Hirao, and Y. Ohishi, J. Phys. Soc. Jpn. 88, 123701 (2019).

[20] V. S. Minkov, V. B. Prakapenka, E. Greenberg, and M. I. Eremets, Angew. Chemie Int. Ed. 59, 1 (2020).

[21] Y. Li, L. Wang, H. Liu, Y. Zhang, J. Hao, C. J. Pickard, J. R. Nelson, R. J. Needs, W. Li, Y. Huang, I. Errea, M. Calandra, F. Mauri, and Y. Ma, Phys. Rev. B 93, 020103(R) (2016).

[22] T. T. Cui, D. Chen, J. C. Li, W. Gao, and Q. Jiang, New J. Phys. 21, 033023 (2019).

[23] I. Troyan, A. Gavriliuk, R. Ruffer, A. Chumakov, A. Mironovich, I. Lyubutin, D. Perekalin, A. P. Drozdov, and M. I. Eremets, Science 351, 1303 (2016).

[24] F. Capitani, B. Langerome, J.-B. Brubach, P. Roy, A. Drozdov, M. I. Eremets, E. J. Nicol, J. P. Carbotte, and T. Timusk, Nat. Phys. 13, 859 (2017).

[25] A. O. Lyakhov, A. R. Oganov, H. T. Stokes, and Q. Zhu, Comput. Phys. Commun. 184, 1172 (2013).

[26] Y. Wang, J. Lv, L. Zhu, and Y. Ma, Comput. Phys. Commun. 183, 2063 (2012). 
[27] C. J. Pickard and R. J. Needs, J. Phys.: Condens. Matter 23, 053201 (2011).

[28] E. Bykova, Single-crystal x-ray diffraction at extreme conditions in mineral physics and material sciences, Ph.D. thesis, University of Bayreuth, 2015.

[29] N. Dubrovinskaia and L. Dubrovinsky, Phys. Scr. 93, 062501 (2018).

[30] T. Meier, F. Trybel, S. Khandarkhaeva, G. Steinle-Neumann, S. Chariton, T. Fedotenko, S. Petitgirard, M. Hanfland, K. Glazyrin, N. Dubrovinskaia, and L. Dubrovinsky, Phys. Rev. X 9, 031008 (2019).

[31] T. Fedotenko, L. Dubrovinsky, G. Aprilis, E. Koemets, A. Snigirev, I. Snigireva, A. Barannikov, P. Ershov, F. Cova, M. Hanfland, and N. Dubrovinskaia, Rev. Sci. Instrum. 90, 104501 (2019).

[32] M. Mezouar, R. Giampaoli, G. Garbarino, I. Kantor, A. Dewaele, G. Weck, S. Boccato, V. Svitlyk, A. D. Rosa, R. Torchio, O. Mathon, O. Hignette, and S. Bauchau, High Press. Res. 37, 170 (2017).

[33] Y. Akahama and H. Kawamura, J. Phys. Conf. Ser. 215, 012195 (2010).

[34] S. Anzellini, A. Dewaele, F. Occelli, P. Loubeyre, and M. Mezouar, J. Appl. Phys. 115, 043511 (2014).

[35] D. Laniel, B. Winkler, E. Koemets, T. Fedotenko, M. Bykov, E. Bykova, L. Dubrovinsky, and N. Dubrovinskaia, Nat. Commun. 10, 4515 (2019).

[36] D. Laniel, M. Bykov, T. Fedotenko, A. V. Ponomareva, I. A. Abrikosov, K. Glazyrin, V. Svitlyk, L. Dubrovinsky, and N. Dubrovinskaia, Inorg. Chem. 58, 9195 (2019).

[37] D. Laniel, B. Winkler, T. Fedotenko, A. Pakhomova, S. Chariton, V. Milman, V. Prakapenka, L. Dubrovinsky, and N. Dubrovinskaia, Phys. Rev. Lett. 124, 216001 (2020).

[38] See Supplemental Material at http://link.aps.org/supplemental/ 10.1103/PhysRevB.102.134109 for the full description of the experimental and theoretical methods, the full crystallographic details on all structures solved by single-crystal $\mathrm{x}$-ray diffraction, as well as complementary figures.
[39] S. J. Clark, M. D. Segall, C. J. Pickard, P. J. Hasnip, M. I. J. Probert, K. Refson, and M. C. Payne, Z. Kristallogr. - Cryst. Mater. 220, 567 (2005).

[40] J. P. Perdew, K. Burke, and M. Ernzerhof, Phys. Rev. Lett. 77, 3865 (1996).

[41] K. Lejaeghere, G. Bihlmayer, T. Bjorkman, P. Blaha, S. Blugel, V. Blum, D. Caliste, I. E. Castelli, S. J. Clark, A. Dal Corso, S. de Gironcoli, T. Deutsch, J. K. Dewhurst, I. Di Marco, C. Draxl, M. Duak, O. Eriksson, J. A. Flores-Livas, K. F. Garrity, L. Genovese et al., Science 351, aad3000 (2016).

[42] H. J. Monkhorst and J. D. Pack, Phys. Rev. B 13, 5188 (1976).

[43] S. M. Arveson, Y. Meng, J. Lee, and K. K. M. Lee, Phys. Rev. B 100, 054106 (2019).

[44] O. Degtyareva, E. Gregoryanz, M. Somayazulu, P. Dera, H. O. K. Mao, and R. J. Hemley, Nat. Mater. 4, 152 (2005).

[45] V. Milman and B. Winkler, Z. Kristallogr. - Cryst. Mater. 216, 99 (2001).

[46] T. A. Strobel, P. Ganesh, M. Somayazulu, P. R. C. Kent, and R. J. Hemley, Phys. Rev. Lett. 107, 255503 (2011).

[47] H. Fujihisa, H. Yamawaki, M. Sakashita, K. Aoki, S. Sasaki, and H. Shimizu, Phys. Rev. B 57, 2651 (1998).

[48] C. Hejny, L. F. Lundegaard, S. Falconi, M. I. McMahon, and M. Hanfland, Phys. Rev. B 71, 020101 (2005).

[49] H. Luo, R. G. Greene, and A. L. Ruoff, Phys. Rev. Lett. 71, 2943 (1993).

[50] O. Degtyareva, E. Gregoryanz, H. K. Mao, and R. J. Hemley, High Press. Res. 25, 17 (2005).

[51] C. J. Pickard and R. J. Needs, Nat. Phys. 3, 473 (2007).

[52] E. J. Pace, X.-D. Liu, P. Dalladay-Simpson, J. Binns, M. PeñaAlvarez, J. P. Attfield, R. T. Howie, and E. Gregoryanz, Phys. Rev. B 101, 174511 (2020).

[53] D. Duan, Y. Liu, F. Tian, D. Li, X. Huang, Z. Zhao, H. Yu, B. Liu, W. Tian, and T. Cui, Sci. Rep. 4, 6968 (2015).

[54] H. Fujihisa, H. Yamawaki, M. Sakashita, A. Nakayama, T. Yamada, and K. Aoki, Phys. Rev. B 69, 214102 (2004).

[55] E. E. Gordon, K. Xu, H. Xiang, A. Bussmann-Holder, R. K. Kremer, A. Simon, J. Köhler, and M. H. Whangbo, Angew. Chem. Int. Ed. 55, 3682 (2016). 